\title{
Explorative screening of potential peptide activities generated by plant-based proteins after in vitro simulation of static digestion
}

\section{Abstract \\ Introduction}

The gastrointestinal digestion of food proteins can generate peptides having a wide range of biological activities and possibly involved in many physiological processes. In this study, we screened various potential bioactivities generated by plant-based protein samples as compared to one animal-based protein after in vitro simulation of gastrointestinal digestion (SGID).

\section{Materials and methods}

1 protein samples: Whey protein (WhP) as an animal protein reference (tested twice, in each set), 3 grades of pea protein (PeaP1, PeaP2 and PeaP3) and an hydrolyzed pea protein (HPeaP), pea and wheat albumins (PeaA and WA), wheat protein (WP), potato, fava bean and oat proteins (PP, FBP and OP) were submitted to SGID.

Undigested and digested protein samples were then tested in different in vitro, cellular or acellular models, including measures on : 1/energy homeostasis through their ability to promote satiety hormones (CCK and GLP-1) secretion by STC-1 cells, to inhibit DPP-IV activity and to interact with opioid receptors; 2/anti-hypertensive properties through their ability to inhibit ACE activity; 3/antiinflammatory properties through their ability to decrease IL-8 secretion by Caco-2 cells submitted or not to LPS; 4/antioxidant properties through their ability to inhibit production of the 3 reactive oxygen species ( $\mathrm{ROS}$ ): $\mathrm{O}_{2}^{-}, \mathrm{H}_{2} \mathrm{O}_{2}$ and $\mathrm{HO}^{-}$.

\section{Results}

The SGID of the 11 protein samples led to bioactive peptides able to stimulate CCK and GLP-1 secretion by enteroendocrine cells in a dose-dependent manner, to inhibit in vitro DPP-IV and ACE activity and to bind opioid receptors. They were also able to decrease production of ROS. Anyway, no anti-inflammatory impact through inhibition of IL-8 secretion has been highlighted in this study but no pro-inflammatory impact was detected neither; even more, some protein samples lost their pro-inflammatory potential after digestion. The best candidates answering globally to the main activities explored were PeaP1, PeaP2, HPeaP, PP and FBP with some specificities.

\section{Discussion}

These protein samples could thus be potentially valorized in the future in the context of type 2 diabetes and related cardiovascular risk prevention, or for their antioxidant properties in the context of cardiovascular diseases or ageing. Moreover, those peptides could offer natural preventive alternatives to some drugs. This will need further exploration and in vivo validation.

\section{Conflict of Interest}

Protein samples and prototypes have been provided by Roquette. Catherine Lefranc-Millot, Laetitia Guérin-Deremaux and Juliette Caron are employed by Roquette. 